\title{
On the Neglect of Local Coulomb Interaction on Oxygens in Perovskites Described by the Multiband $d-p$ Model
}

\author{
K. Rościszewski And A.M. Oleś \\ M. Smoluchowski Institute of Physics, Jagiellonian University, prof. S. Łojasiewicza 11, PL-30348 Kraków, Poland
}

\begin{abstract}
On the example of $\mathrm{TiO}_{4}$ layer (such as realized in $\mathrm{Sr}_{2} \mathrm{TiO}_{4}$ ) we study electronic structure of multiband $d-p$ models describing transition metal perovskites. As suggested by experiment, the studied system is predicted to be a robust nonmagnetic insulator. A realistic treatment of electronic structure requires one to introduce non-zero Coulomb local interactions at $2 p$ oxygen orbitals. However, up till now majority of papers based upon multiband models made an approximation of neglecting such interactions. We show that this simplification does not lead to serious problems in predictions of the electronic structure provided the Coulomb interactions at titanium ions and charge transfer gap are suitably renormalized (so they become entirely different with respect to the true microscopic $d-p$ model parameters).
\end{abstract}

DOI: 10.12693/APhysPolA.133.356

PACS/topics: 71.15.-m, 75.25.Dk, 75.47.Lx

\section{Introduction}

Shortly after the discovery of high temperature superconductors, it was realized that both copper $3 d$ and oxygen $2 p$ orbitals contribute to the correlated states in the cuprates and should be thus included explicitly in the respective multi-band $d-p$ model for $\mathrm{CuO}_{2}$ planes $[1,2]$ or $\mathrm{CuO}_{3}$ chains [3]. Nowadays a common approach is to use $d-p$ model for the description of electronic structure of a correlated insulator for the analysis of charge and magnetization density distribution and electron correlations, exactly as was done in cuprates in the past [4]. The multi-band $d-p$ model is richer and provides one with a much more wide physical picture than simpler models which feature only $d$-orbitals $[5,6]$. Quite recently the multi-band $d-p$ models featuring on-site interactions defined both on oxygens and on metal ions were investigated for ruthenium, iridium, and titanium oxides $[7,8]$. We focused on the full description of Coulomb interactions including oxygen orbitals and showed that they cannot be neglected. In contrast to this many papers in the literature neglect such interactions on oxygens. We will show that this simplification does not lead to serious problems in prediction of the electronic structure provided Coulomb interactions at titanium ions and chargetransfer-gap are properly renormalized.

In the previous paper [8] the preliminary density functional theory computations were performed on quasi-twodimensional $\mathrm{Sr}_{2} \mathrm{TiO}_{4}$ and it was found that the ionicity of $\mathrm{Sr}$ ions is exactly +2 . Therefore for the description of the two-dimensional (2D) model for $\mathrm{TiO}_{4}$ layer one must assume that the number of valence electrons per $\mathrm{TiO}_{4}$ unit is exactly 24 . This number is used as input to unrestricted HF computations within multi-band $d-p$

corresponding author; e-mail:

krzysztof .rosciszewski@uj.edu.pl model featuring on-site Coulomb interactions both on titanium ions and oxygen ions. The result is that $\mathrm{Sr}_{2} \mathrm{TiO}_{4}$ is a robust non-magnetic insulator. The experimental band gap of $3.8 \mathrm{eV}$ was recovered from the computations for: $U_{d}=9.0 \mathrm{eV}, U_{p}=4.4 \mathrm{eV}$ and charge-transfer gap $\Delta=\varepsilon_{d}-\varepsilon_{p}=6.5 \mathrm{eV}$ (defined for bare energy levels) [8]. The $d$-electron count on Ti ion is $n_{d} \simeq 1.2$, almost equally distributed over $e_{g}$ and $t_{2 g}$ orbitals.

Following preliminary results from ref. [8] we provide definite prescription how to modify local Coulomb interaction on metal ions and charge-transfer-gap in simplified $d-p$ models which neglect local interations on oxygens.

\section{Multi-band $d-p$ model Hamiltonian}

The multi-band $d-p$ Hamiltonian for $\mathrm{TiO}_{4}$ layer includes five $3 d$ orbitals at each titanium ion and three $2 p$ orbitals at each oxygen ion. It consists of the following parts:

$$
\mathcal{H}=H_{\text {kin }}+H_{\text {diag }}+H_{\text {int }},
$$

where the different terms in Eq. (1) stand for the kinetic energy $\left(H_{\text {kin }}\right)$, crystal-field splitting $\left(H_{\text {diag }}\right)$, and the intraatomic Coulomb interactions $\left(H_{\text {int }}\right)$.

The kinetic (hopping) part of the Hamiltonian is:

$$
H_{\text {kin }}=\sum_{\{i, \mu ; j, \nu\}, \sigma} t_{i, \mu ; j, \nu} c_{i, \mu, \sigma}^{\dagger} c_{j, \nu, \sigma}+\text { H.c., }
$$

where we employ notation with $c_{j, \nu, \sigma}^{\dagger}$ standing for the creation of an electron at site $j$ in an orbital $\nu$ with up and down spin, $\sigma=\uparrow, \downarrow$. The model includes all $d$ orbital states on titanium, $\nu \in\left\{x y, y z, z x, 3 z^{2}-r^{2}, x^{2}-y^{2}\right\}$, and three $2 p$ orbitals per oxygen atom, $\nu \in\left\{p_{x}, p_{y}, p_{z}\right\}$. Alternatively, i.e., choosing a more intuitive notation, we can write $d_{j, \nu, \sigma}^{\dagger}$ for $d$ orbitals, while $p_{j, \nu, \sigma}^{\dagger}$ for $p$ orbitals. The matrix elements $t_{i, \mu ; j, \nu}$ are non-zero only for nearestneighbor atoms; they are obtained using Slater-Koster rules $[7,9]$.

The $H_{\text {diag }}$ depends only on particle operator numbers and describes effects of crystal field and the difference of 
reference energies (i.e., charge-transfer-gap),

$$
\Delta=\varepsilon_{d}-\varepsilon_{p},
$$

between empty $d$ and empty $p$ orbitals. In the following we fix reference energy at $\varepsilon_{d}=0$, hence instead of $\Delta$ there is only $\varepsilon_{p}$ present in $H_{\text {diag }}$ :

$$
\begin{gathered}
H_{\text {diag }}=\sum_{i, \mu=x, y, z ; \sigma} \varepsilon_{p}(i) p_{i, \mu, \sigma}^{\dagger} p_{i, \mu, \sigma} \\
\quad+\sum_{i, \mu=x y, y z, \ldots ; \sigma}^{c r} f_{\mu, \sigma}^{\dagger} d_{i, \mu, \sigma} d_{i, \mu, \sigma} .
\end{gathered}
$$

Note that the first sum is restricted to oxygen sites, while the second one runs over titanium sites.

The local Coulomb interaction for titantium ions is

$$
\begin{aligned}
& H_{\text {int }}(d)=U_{d} \sum_{m, \mu} n_{m, \mu, \uparrow} n_{m, \mu, \downarrow} \\
& \quad+\frac{1}{2} \sum_{m, \mu \neq \nu}\left(U_{d}-\frac{5}{2} J_{d, \mu \nu}\right) n_{m, \mu} n_{m, \nu} \\
& \quad-\sum_{m, \mu \neq \nu}^{m} J_{d, \mu \nu} \mathbf{S}_{m, \mu} \cdot \mathbf{S}_{m, \nu} \\
& \quad+\sum_{m, \mu \neq \nu}^{m} J_{d, \mu \nu} d_{m, \mu, \uparrow}^{\dagger} d_{m, \mu, \downarrow}^{\dagger} d_{m, \nu, \downarrow} d_{m, \nu, \uparrow},
\end{aligned}
$$

where again $\mu, \nu$ enumerate $d$-orbitals, and $J_{d, \mu \nu}$ is the tensor of on-site interorbital exchange elements [10]). In our computations the whole anisotropic tensor $J_{d, \mu \nu}$ is treated without any simplifications. The formula for local Coulomb interactions at oxygen sites (for $2 p$ orbitals) is analogous.

The effective $d-p$ model requires a number of parameters. The in-plane hopping elements were fixed as $(p d \sigma)=-2.4 \mathrm{eV}$ and $(p d \pi)=1.3 \mathrm{eV}$ and also $(p p \sigma)=$ $0.6 \mathrm{eV}$ and $(p p \pi)=-0.15 \mathrm{eV}$ [11-13]. The choice of the Coulomb elements in $d-p$ model is difficult. There are reliable estimates for $U_{d}(\sim 4 \mathrm{eV})$ but only in effective models featuring solely composite $d$-type Wannier orbitals (i.e., explicit treatment of $p$-orbitals is absent). The typical parameter $U_{d} \sim 4 \mathrm{eV}$ (for titanium ions) is different from that which should be used in the framework of the multiband $d-p$ model. Namely, it is smaller in the $d$-orbital-only model from that used in the $d-p$ model by $\sim 50 \%$ due to a massive screening.

We decided to study several possibilities, namely $U_{d}=$ $4,6,8,9$, and $10 \mathrm{eV}$ according to the data in the literature [14]: $\left(U_{d} \in[7,8] \mathrm{eV}\right)$ [15-17]; $\left(U_{d} \sim 8 \mathrm{eV}\right)$; and $\left.U_{d} \sim 6 \mathrm{eV}[18,19]\right)$. The Hund exchange elements are less screened than intraorbital Coulomb elements and are closer to their atomic values. For the Hund exchange $J_{d}$ (between two $t_{2 g}$ electrons) and for our computations we decided to select $J_{d}=0.8 \mathrm{eV}$ according to refs. $[19,20]$ (also other possibilities were studied, namely $J_{d}=0.9 \mathrm{eV}$ and $J_{d}=1.0 \mathrm{eV}$ ). The Coulomb repulsion $U_{p}$ for oxygen $p$-orbitals was fixed as $U_{p}=4.4 \mathrm{eV}$ like in refs. [7, 21, 22], while the Hund exchange was fixed as $J_{p}=0.8 \mathrm{eV}[20]$. Next the average $t_{2 g}$ to $e_{g}$ crystal-field splitting (for bare levels) which we studied were either $2.0 \mathrm{eV}$ or $3.0 \mathrm{eV}[12,23,24]$. Finally, very important parameter for the charge distribution in $\left(\mathrm{TiO}_{2}\right)^{2-}$ planes is a charge-transfer gap $\Delta$ (3) which was examined as a continuous parameter in the range from $1.0 \mathrm{eV}$ up to $9.0 \mathrm{eV}$.

We used the unrestricted HF approximation to investigate the $\mathrm{TiO}_{4}$ cluster $\left(4 \times 4 \mathrm{TiO}_{4}\right.$ units with cyclic boundary conditions) and with $N_{e}=24$ electrons per single $\mathrm{TiO}_{4}$ unit. The implementation of the model Hamiltonian (1) is the same as described in refs. $[7,11,25,26]$. The possibilities of different types of order were taken into account: nonmagnetic phase, ferromagnetic and antiferromagnetic with spins aligned along: $(1,1,0)$ or $(1,0,0)$ or $(0,0,1)$ direction. We performed runs for all combinations of the Hamiltonian parameters. Any reasonable parameter set leads to nonmagnetic insulator as the ground state. The experimental band gap in $\mathrm{Sr}_{2} \mathrm{TiO}_{4}$ is $3.8 \mathrm{eV} \mathrm{[27]} \mathrm{and} \mathrm{it} \mathrm{is} \mathrm{reproduced} \mathrm{when} \mathrm{the} \mathrm{Hamilto-}$ nian parameters are properly tuned to $U_{d}=9.0 \mathrm{eV}$, $J_{d}=0.8 \mathrm{eV}$ and $\Delta \simeq 6.5 \mathrm{eV}[8]$.

\section{Consequences of the neglect of Coulomb repulsion on oxygens}

Let us now study what happens when Coulomb repulsion on oxygens is neglected. We argue that in general it is not correct to set $U_{p}=J_{p}=0$ but this approximation/simplification was used by almost all the authors up to now.

Thus, we performed multiple HF computations using Hamiltonian parameter sets with $U_{p}=J_{p}=0$ and with numerous differently renormalized $U_{d}$ and $\Delta$. Much to our surprise we obtained quite normal electronic structure with nonmagnetic ground state as a generic solution. When studying various possibilities how to renormalize $U_{d}$ and $\Delta$ so as the obtained results are physically sensible we obviously should look after such sets of $U_{d}$ and $\Delta$ which reproduce experimental band gap of $3.8 \mathrm{eV}$. The results coming out from this approach are shown in Fig. 1.

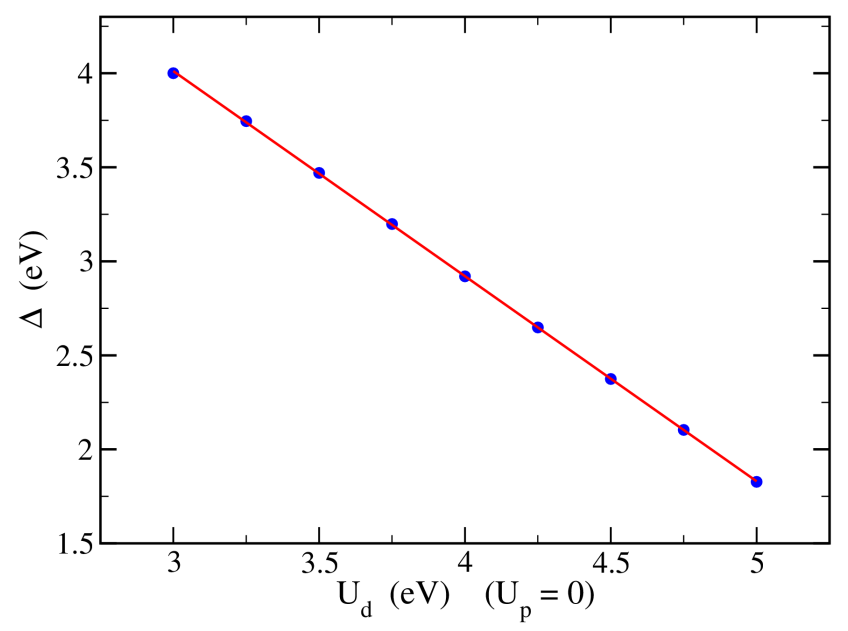

Fig. 1. Charge-transfer energy $\Delta(3)$ versus $U_{d}$ for the parameters which reproduce experimental band gap of $3.8 \mathrm{eV}$. The extrapolated dependence is linear $-\Delta \approx$ 7.28-1.09 $U_{d}$ (with $U_{d}$ in eV). Fixed parameters of the $d$ - $p$ Hamiltonian are: $U_{p}=0, J_{0}=0$; crystal field $t_{2 g}$ to $e_{g}$ splitting is $2.0 \mathrm{eV}$ and $J_{d}=0.8 \mathrm{eV}$. 


\section{Renormalization of $U_{d}$ and $\Delta$ for $U_{p}=0$ models}

From Fig. 1 if follows that if one decides to model the titanium perovskite neglecting the local Coulomb repulsion on oxygen $\left(U_{p}=J_{p}=0\right)$ and in addition if one accepts $U_{d}=4 \mathrm{eV}$ then the charge-transfer energy should be chosen as $\Delta \approx 3.0 \mathrm{eV}$. Note that the usual parameters of the $d-p$ multi-band model are by $50 \%$ larger, just to remind: $U_{d}=9 \mathrm{eV}$ and $\Delta=6.5 \mathrm{eV}$.

If a different value of $U_{d}$ is advocated in a particular paper one can pick out from the literature still we can predict the precise value of $\Delta$ which should be appropriate in such the context.

\section{Summary}

We have taken the number of electrons which follows from the ionic configuration of $\mathrm{Sr}^{2+}$ ions for a periodic $4 \times 4 \mathrm{TiO}_{4}$ cluster, as suggested by ab initio electronic structure calculations and examined in electronic distribution within unrestricted HF computations performed using the multi-band $d-p$ model. Several possibilities for Hamiltonian parameters were studied. For any reasonable choice of the parameters we find a good nonmagnetic insulator, with a gap reproduced for $U_{d}=9.0 \mathrm{eV}$, $U_{p}=4.4 \mathrm{eV}$, and a large charge-transfer gap $\Delta=6.5 \mathrm{eV}$.

We also considered a simplified set of parameters with $U_{p}=J_{p}=0$ and find that this choice implies considerable reduction of the remaining parameters: $(i)$ the value of $U_{d}$ is smaller by $\sim 50 \%$; $(i i)$ the charge-transfer gap $\Delta$ is also reduced to $\sim 3.0 \mathrm{eV}$. Only taking these renormalization one can reproduce the essential features of the electronic structure of $\mathrm{Sr}_{2} \mathrm{TiO}_{4}$. We remark that while such a renormalization is possible for the bulk, the treatment of systems with non-equivalent oxygen and transition metal positions would require a complete parameter set with finite electron interactions at oxygen $2 p$ orbitals.

\section{Acknowledgments}

We kindly acknowledge support by Narodowe Centrum Nauki (NCN) under Project No. 2012/04/A/ST3/00331.

\section{References}

[1] V.J. Emery, Phys. Rev. Lett. 58, 2794 (1987); C.M. Varma, S.S. Schmitt-Rink, E.E. Abrahams, Solid State Commun. 62, 681 (1987); A.M. Oleś, J. Zaanen, P. Fulde, Physica B $+C$ 148, 260 (1987).

[2] E. Arrigoni, M. Aichhorn, M. Daghofer, W. Hanke, New J. Phys. 11, 055066 (2009).

[3] A.M. Oleś, W. Grzelka, Phys. Rev. B 44, 9531 (1991).

[4] J.B. Grant, A.K. McMahan, Phys. Rev. B 46, 8440 (1992).

[5] M. Möller, G.A. Sawatzky, M. Berciu, Phys. Rev. Lett. 108, 216403 (2012); Phys. Rev. B 86, 075128 (2012).

[6] K. Bieniasz, A.M. Oleś, Phys. Rev. B 88, 115132 (2013).
[7] K. Rościszewski, A.M. Oleś, Phys. Rev. B 91, 155137 (2015); Phys. Rev. B 93, 085106 (2016).

[8] K. Rościszewski, P. Piekarz, A.M. Oleś, Physica Status Solidi (b) 254, 1700022 (2017).

[9] C. Slater, G.F. Koster, Phys. Rev. 94, 1498 (1954).

[10] A.M. Oleś, G. Khaliullin, P. Horsch, L.F. Feiner, Phys. Rev. B 72, 214431 (2005).

[11] T. Mizokawa, A. Fujimori, Phys. Rev. B 54, 5368 (1996).

[12] M. Mochizuki, J. Phys. Soc. Jpn. 71, 2039 (2002); M. Mochizuki, M. Imada, J. Phys. Soc. Jpn. $\mathbf{7 3}$ 1833 (2004); New J. Phys. 6, 154 (2004).

[13] H. Wadati, A. Chikamatsu, M. Takizawa, H. Kumigashira, T. Yoshida, T. Mizokawa, A. Fujimori, M. Oshima, N. Hamada, J. Phys. Soc. Jpn. 78, 094709 (2009).

[14] A.I. Lichtenstein, V.I. Anisimov, M.I. Katsnelson, Electronic structure of transition metal oxides, in: Electronic Structure and Magnetism of Complex Materials, Eds. D.J. Singh, D.A. Papaconstantopoulos, Springer-Verlag, Berlin, Heidelberg 2003).

[15] P.V. Ong, J. Lee, W.E. Pickett, Phys. Rev. B 83 193106 (2011).

[16] S.G. Park, B. Magyari-Köpe, Y. Nishi, Phys. Rev. B 82, 115109 (2010)

[17] B. Magyari-Köpe, S.G. Park, H. Lee, Y. Nishi, J. Mater. Sci. 47, 7498 (2012).

[18] M.W. Haverkort, M. Zwierzycki, O.K. Andersen, Phys. Rev. B 85, 165113 (2012).

[19] V.I. Anisimov, J. Zaanen, O.K. Andersen, Phys. Rev.B 44, 943 (1991)

[20] S.V. Streltsov, A.S. Mylnikova, A.O. Shorikov, Z.V. Pchelkina, D.I. Khomskii, V.I. Anisimov, Phys. Rev. B 71, 245114 (2005).

[21] M.S. Hybertsen, M. Schlüter, N.E. Christensen, Phys. Rev. B 39, 9028 (1989).

[22] K. Wohlfeld, S. Nishimoto, M.W. Haverkort, J. van den Brink, Phys. Rev. B 88, 195138 (2013).

[23] H. Ikeno, F.M.F. de Groot, E. Stavitski, I. Tanaka, J. Phys.: Condens. Matter 21, 104208 (2009); H. Ikeno, H. Mizoguchi, I. Tanaka, Phys. Rev. B 83, 155107 (2011).

[24] Z. Ke-Jin, M. Radovic, J. Schlappa, V. Strocov, R. Frison, J. Mesot, K. Patthey, T. Schmitt, Phys. Rev. B 83, 201402(R) (2011).

[25] T. Mizokawa, L.H. Tjeng, G.A. Sawatzky, G. Ghiringhelli, O. Tjernberg, N.B. Brookes, H. Fukazawa, S. Nakatsuji, Y. Maeno, Phys. Rev. Lett. 87, 077202 (2001).

[26] A. Avella, P. Horsch, A.M. Oleś, Phys. Rev. B 87 , 045132 (2013); A. Avella, A.M. Oleś, P. Horsch, Phys. Rev. Lett. 115, 206403 (2015).

[27] J. Matsuno, Y. Okimoto, M. Kawasaki, Y. Tokura, Phys. Rev. Lett. 95, 176404 (2005). 